\title{
Gallium Nitride Nanotube and its Application as Transistors
}

\author{
Bagavathi Chandrasekara \\ Amrita School of Engineering \\ Coimbatore, India
}

\author{
K A Narayanankutty \\ Amrita School of Engineering \\ Coimbatore, India
}

\begin{abstract}
In search of opto-electronic nano materials, we often come across Gallium Nitride nanotubes (GaN-NT) with excellent electrical and optical characteristics. Gallium Nitride nanotubes are predominantly semiconducting and have been less explored in its application as a transistor channel through Density Functional Theory (DFT). Comparing Gallium Nitride nanotubes with Boron Nitride nanotubes (BN-NT) and Carbon nanotubes (CNT), we have obtained distinguishing features of Gallium Nitride nanotubes. In this work, Transistor simulation with Gallium Nitride nanotubes has been reported with the nanotube as channel. Properties of various configurations of nanotubes are compared among Carbon, Boron Nitride and Gallium Nitride nanotubes.
\end{abstract}

\section{General Terms}

Nanotube Transistor Modeling

\section{Keywords}

Gallium Nitride nanotubes, GaN nanotube transistor, Density Functional Theory (DFT), bond rotation, Boron Nitride nanotubes

\section{INTRODUCTION}

Recently it has been claimed the semiconductor size limit has been reached for silicon [1]. Nanotubes devices outweigh the performance of Silicon devices. A group of IBM researchers have synthesized a 9-nm nanotube transistor which has been found to work efficiently compared to any other transistor of that size [2]. Nanotubes other than organic origin can also be used as a transistor channel. Many such nanotubes like Boron Nitride nanotube, Gallium Nitride, Silica, Nickel Halide are available [3]. Our subject of interest would be Gallium Nitride nanotubes and transistors with them as channels. Gallium Nitride $(\mathrm{GaN})$ in bulk had established itself as a wonderful material with excellent applications in high power applications, LED applications and high mobility applications. Gallium Nitride differs from other materials as it has a stable hexagonal structure [4]. GaN bulk transistors have shown to be lightning-fast and allow frequencies of up to $205 \mathrm{GHz}$ which would make the current devices faster and economical [5]. Without loss of generality, Gallium Nitride nanotubes (GaN-NT) also had been found to have very good electrical and optical properties.

Single crystal Gallium Nitride nanotubes have been synthesized successfully using epitaxial casting method [6]. What known less about them are their applications as devices like transistors. Till now there has been literally very little research in the area of Gallium Nitride nanotube transistors. Following the path of Carbon nanotubes (CNT), Gallium Nitride nanotubes can also be used to obtain field effect transistors. The predecessor for Gallium Nitride nanotubes is the Boron Nitride nanotubes (BN-NT) which also have been synthesized and have been found to have semiconducting properties [7]. Even though both Boron Nitride and Gallium Nitride nanotubes belong to the same class of III-group Nitride nanotubes, there are differentiating features between them [8]. From simulation studies through Density Functional Theory concepts, it has been observed that, Boron Nitride and Carbon nanotubes behave in a similar manner. The energy of Gallium Nitride nanotubes, which decides the stability of nanotubes, depends on the chiral factor. The armchair configurations have been found to have lower energy compared other configurations. The bandgap of the nanotubes also depend on chirality [9]. Spin effects on the nanotubes are also considered for analysis. On the mechanical perspective of nanotubes, selective single bond rotation effects have been considered [10]. Transistor simulation of Gallium Nitride nanotube has been proposed.

In this paper, a basic introduction about density functional theory is provided. Gallium Nitride nanotubes of various chiral configurations are tried for their properties using simulation done using Atomistix Tool Kit [11] software based on the principles of Density Functional Theory. The comparison of Carbon, Boron Nitride and Gallium Nitride nanotubes has been presented in this work. A comparison of properties of all the III-group Nitride nanotubes is reported.

\section{DENSITY FUNCTIONAL THEORY}

All material Density Functional Theory utilizes the basic task played by electron density in determining the properties of materials [12]. Even after applying many simplifications to many-body Schrodinger problem through approximations, it still remains complex. Complex nature of many-body problem results from the presence of large number of electrons and their indifferent interactions among themselves known as electron-electron interactions. Even with a two electron system, many times it is difficult to solve. It becomes still more complex when an external potential is present in the system. The computational cost of this problem remains high. By using DFT, the complexity of wave function approach can be reduced. The DFT uses the electron density $\mathrm{n}(\mathrm{r})$ as a fundamental entity to use it as a functional for the properties such as energy instead of wave functions.

When Schrodinger equation is solved for many-body problem for Hamiltonian and corresponding eigen states, it is possible to calculate expectation value of any operator [1]. Hamiltonian is the operator associated with the total energy of the system. In case of an external potential $\mathrm{V}_{\text {ext }}(\mathrm{r})$, energy due to external potential along with default terms, kinetic (T) and potential energy $(\mathrm{V})$, should be included. The Hamiltonian,

$$
H=T+V+V_{e x t}
$$

If Hamiltonian of a system is known, according to DFT, it is possible to find the ground state density of the system. Since 
other components (kinetic and potential) of Hamiltonian remains constant, only external potential determines the electron density. This phenomenon is the basis for Hohenberg-Kohn theorems. Hohenberg-Kohn theorems were used to simply the DFT to be an exact formulation of manybody problem. For a system of electrons in an external potential $\mathrm{V}_{\text {ext }}(\mathrm{r})$, the potential is exactly determined by ground state electron density [13]. If system Hamiltonian is obtained from electron density, then the system is declared as completely defined.

Hohenberg-Kohn theorem mentions to use electron density as the basic entity to obtain any parameter from the system. But it didn't mention any method for obtaining it. Kohn-Sham formulation [14] provides a method to use a reference (noninteracting) system and to search for an external potential whose ground state density would be same as real system [1]. The equations have to be solved self-consistently to obtain the exact density and potential for the real system.

The self consistent field formulation is performed to get exact electron density and the corresponding effective potential U. The iteration is started with an initial guess of electron density $\mathrm{n}(\mathrm{r})$. The number of electrons in a level will be given by Fermi function instead of an integer. $U_{\text {new }}$ is the new effective Kohn Sham potential after iteration and $\mathrm{N}_{0}$ is the number of electrons under equilibrium condition of the system and $\mathrm{N}$ is number of electrons in the system at any time.

$$
N=\int n(r) d r
$$

The rate of electrons escaping from the terminals to contacts is called escape rate $(\gamma)$. The escape rate of source and drain are given as $\gamma_{1}$ and $\gamma_{2}$. These escape rates determine the rate of flow of electrons and electron density in the device. The iteration stops when there is no change in the effective potential value.

When Schrodinger equation is solved for the Hamiltonian and many-body eigen states, expectation value of any operator can be obtained. The Hamiltonian, therefore, determines the expectation value of any operator, and, in particular, the Hamiltonian determines the ground-state density, since this is just the ground-state expectation value of the density operator [1].

Even though simplifications had been made for the wave function crisis, the problem of electron-electron interaction still remains. Electrons interact among themselves in a very sophisticated way. Approximations are required for the interactions of electrons.

The till-now-best approximation known way is the Local Density Approximation (LDA) [15]. It is known that exchange and correlation effects are local in character and the exchange correlation energy is simply the integral of the exchange correlation energy density at each point in space assumed to be the same as a homogeneous electron gas with that density. Based on this approximation, exchange correlation energy is calculated.

By employing Kohn Sham equations and Local Density Approximation (LDA), the many-electron problem can be simplified as DFT is the only efficient method used for quantum analysis for more than four decades. It gives Successful approximation. Interaction of electrons is given by exchange correlation energy functional

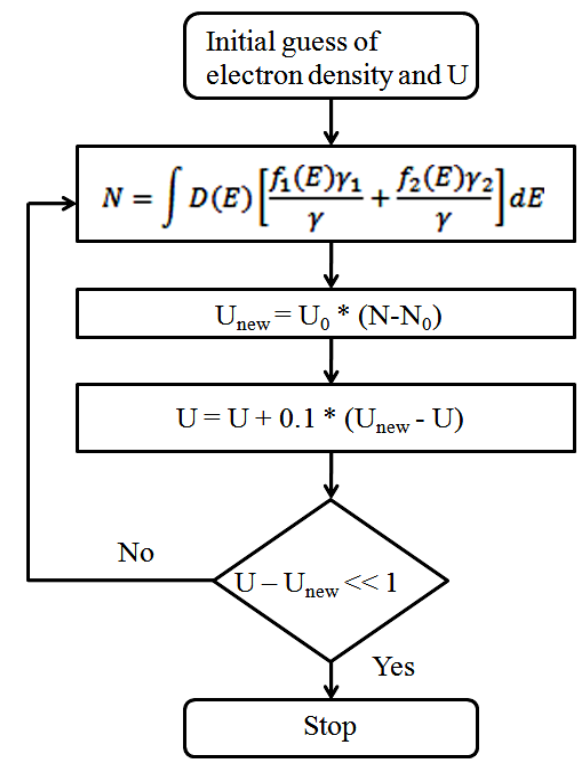

Fig 1. Self-consistent formulation of Kohn-Sham scheme.

\section{GALLIUM NITRIDE NANOTUBES}

Gallium Nitride as a well known material suitable for high power, high density and opto-electronic applications, has been proved of excellent characteristics. Many products such as power supply, inverters and motor drives started to concentrate on Gallium Nitride whose market is expected to be more than a billion dollars within a decade [16]. Another wonder supporting Gallium Nitride growth is that its properties as a nanotube is astonishing. Gallium Nitride nanostructures are of particular interest because it has been shown to yield good quality wires and can be either as-grown $\mathrm{n}$ type or doped $\mathrm{p}$ type by the incorporation of magnesium during growth [17].

Gallium Nitride nanotubes were successfully synthesized by using epitaxial casting method in which Zinc oxide nanorods were used as templates [6] [18]. After this significant invention, there had been a lot of research in this field to understand these nanotubes and put them to efficient use. Some of the properties concentrated in this paper are mechanical and electronic properties. Mechanical properties are important as it decides the actual realization of a device. Electronic properties determine its use in opto electronics, transistor and other high power applications.

Mechanical properties has been investigated based on Molecular Dynamics simulation [19] [10]. Bond rotation analysis is performed by selecting a single bond and rotating through a particular angle $\theta$. For bond rotation studies, consider a single bond along circumferential direction and allows bond rotation through $90^{\circ}$, then the nanotube property changes. If the bond rotated nanotube variation is less compared to default nanotube, then it is mechanically stable. Such bond rotations usually elongate the tube and they are called Stone Wales transformation [10]. A reduction in total energy is expected in case of Gallium Nitride nanotube implying its stability. Bond rotation is expected to cause variation in electronic cloud around the nanotube and change in density of states. When a bond rotation event occurs, there is a deviation from ideal perfect nanotube and as obvious as it seems there should be a reduction in transmission coefficients.

Electronic properties are analyzed based on band structure, eigen states, density of states, electron density, total energy 
and transmission spectrum of the nanotubes. Gallium Nitride nanotubes are inherently semiconducting. They are expected to have high transmission coefficient at high energy values as they are well known for high energy applications in bulk [20]. Eigen states are the orbitals whose eigen values correspond to the energy levels. The occupied eigen states defines the electron density of the system [21].

When compared with Carbon nanotubes, Gallium Nitride nanotubes have discrete eigen states and electron density. Density of states indirectly defines ability of conduction by the device. Higher density of states implies good transmission. Energy is calculated based on density functional theory where the ingredients of total energy are external, electrostatic, kinetic, exchange correlation and entropy terms. External energy is due to any external voltage applied to the nanotube. Exchange correlation energy includes the interaction between electrons in a multi-electron system. Transmission process through the nanotube is processed by the density of energy levels in the channel [22].

\section{GALLIUM NITRIDE NANOTUBE TRANSISTOR}

Transistors are the core devices of microelectronics and Gallium Nitride nanotubes fulfill the basic criterion of a transistor channel as all of them are semiconducting. The essential feature of a well-designed transistor is that the gate is much closer to the semiconducting channel, allowing it to hold the channel potential constant despite the voltage on the drain [21].

Moore's law has been driving the designers to reduce the size of transistors every now and then. After a limit, it has been found various quantum mechanical effects had been affecting the performance of transistors as devices started entering nano regime. The transistors can no longer be applied with classical physics concepts. When moving to such tiny dimensions, quantum concepts are to be applied. Definitions of quantities like resistance, current and conductance have to be seen through a new perspective.

For one-dimensional devices, the conductance of a single level gets defined as a function of density of states and the conductance has been limited to a maximum value of $80 \times 10^{-6}$ $\Omega^{-1}$ [23]. Density of states is determined by the number of energy levels within a range of the Fermi level in the channel of the device. When number of energy levels in these range increases, the interaction of electron that would be present in those levels and the complexity of computation increase. A transistor with nanotubes as channels belongs to onedimensional devices.

To determine the current flow in nano transistor, equilibrium energy level diagram should be used to calculate the electrochemical potential $(\mu)$ and the movement of electrons when an external bias is applied. For the transistor to perform properly, states either filled or empty should be available near the electrochemical potential [21].

In equilibrium condition, source, drain and channel will have same electrochemical potential. When a bias is applied, the levels in source and drain split as $\mu 1$ and $\mu 2$ depending on the applied voltage.

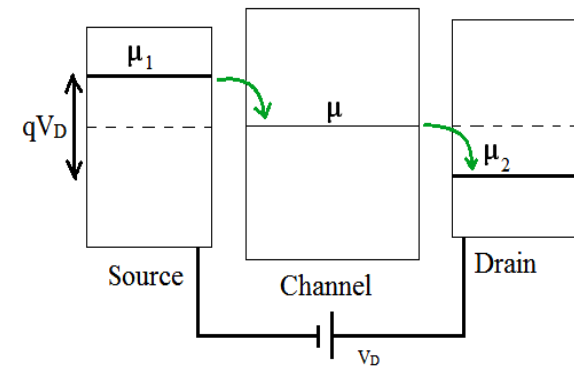

Fig 2. Structure of a nano-level transistor under external bias

Source tries to fill the channel with electrons and drain tries to empty it. Thus the current flows in the circuit. The escape rate of source and drain are given as $\gamma 1$ and $\gamma 2$. The current flow depends on the electrochemical potential of the terminals, escape rate of electrons and external bias. The electrochemical potential of the terminals define the number of electrons in these terminals at any instant. The current can be calculated as follows.

$$
I=\frac{2 \pi q}{h} \int D(E) \frac{\left(f_{1}(E)-f_{2}(E)\right) \gamma_{1} \gamma_{2}}{\gamma} d E
$$

\section{RESULTS AND DISCUSSION}

The results of the work have been presented in this section. Gallium Nitride nanotubes and Gallium Nitride nanotube transistor are analyzed separately

\subsection{Gallium Nitride nanotubes}

From the synthesis of single crystal Gallium Nitride nanotubes, there has been a lot of research on this nanotube in the search of profitable optoelectronic characteristics. Gallium Nitride is a semiconductor both in bulk and nanotube form.

For simulation purpose, Carbon and Boron Nitride nanotubes are chosen with a bond length of $1.42 \AA$ and Gallium Nitride nanotube with bond length of 1.90 Á. Local density approximation method is used for exchange correlation function.

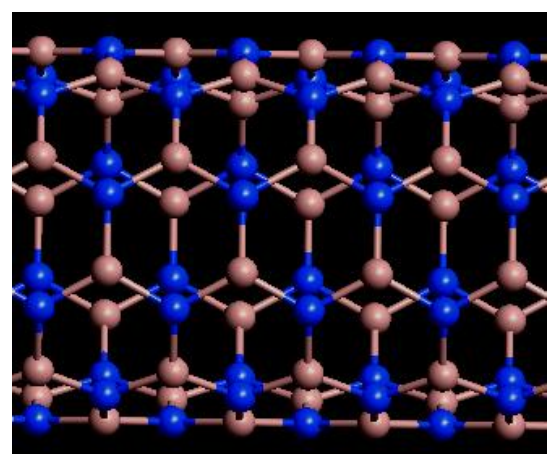

Fig. 3 Gallium Nitride nanotube $(5,5)$ from Virtual Nanolab software

Bond rotation effects can be seen in Gallium Nitride nanotubes as reduction in total energy in Gallium Nitride nanotube is higher compared to reduction in Boron Nitride nanotube. Table 1 shows the change in total energy due to bond rotation. 
Table 1: Energy change due to bond rotation

\begin{tabular}{llll}
\hline & $\begin{array}{l}\text { Total energy } \\
\text { before } \\
\text { rotation (E1) } \\
\text { in eV }\end{array}$ & $\begin{array}{l}\text { Total energy } \\
\text { after } \\
\text { rotation }(\mathrm{E} 2) \\
\text { in eV }\end{array}$ & $\begin{array}{l}\text { Energy } \\
\text { deviation } \\
\Delta \mathrm{E}=\mathrm{E} 1-\mathrm{E} 2 \\
(\mathrm{eV})\end{array}$ \\
\hline $\mathrm{CNT}$ & -15795.38 & -15784.73 & 11 \\
$\mathrm{BN} \mathrm{NT}$ & -17715.02 & -17703.15 & 12 \\
GaN NT & -24976.41 & -24860.36 & 116 \\
\hline
\end{tabular}

There is a random change in density of states of Gallium Nitride nanotube after a bond rotation of $90^{\circ}$ as shown in figure 4 .

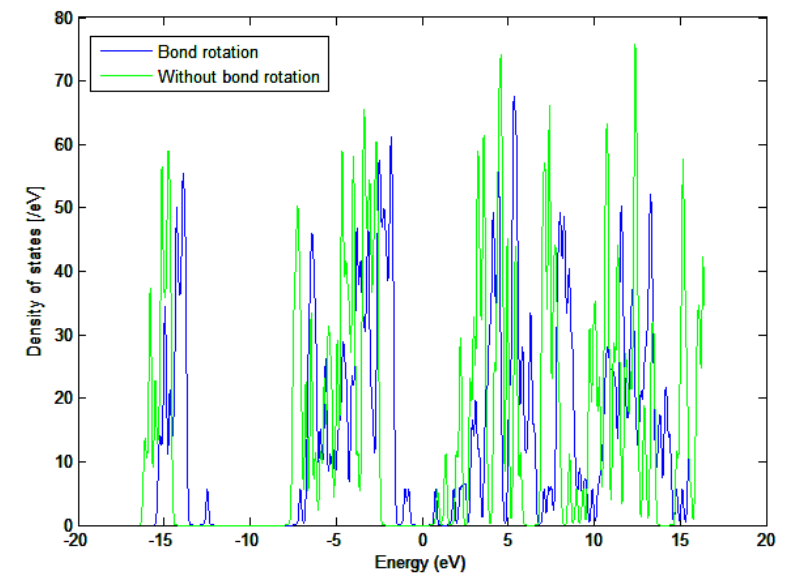

Fig 4. Density of states of Gallium Nitride nanotube before and after bond rotation

The band structure shows all the nanotubes are semiconducting. The band gaps of various GaN nanotubes are shown in figure 5. For nanotubes of same number of atoms in $\mathrm{x}$-direction, the bandgap increases linearly with linear increase in the number of atoms in the y-direction. As number of atoms in the nanotube increases, the bandgap also increases.

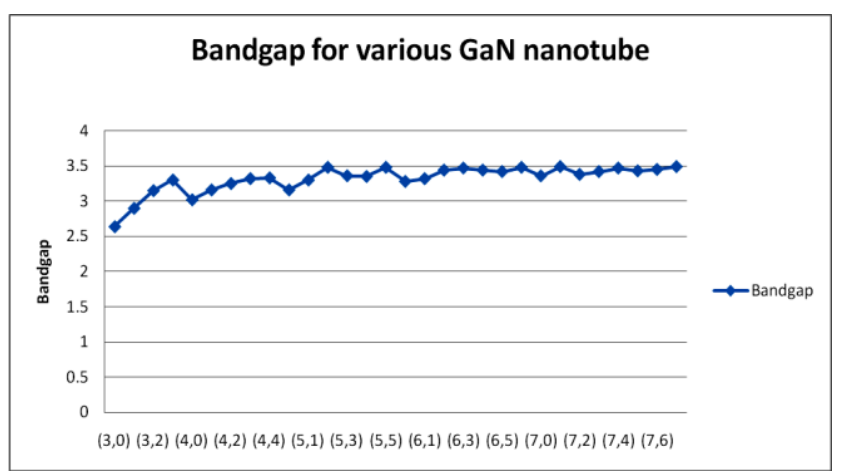

Fig 5. Band gaps of various Gallium Nitride nanotubes

Density of states is found in both sides of the Fermi level predominantly on the positive side of the Fermi level.

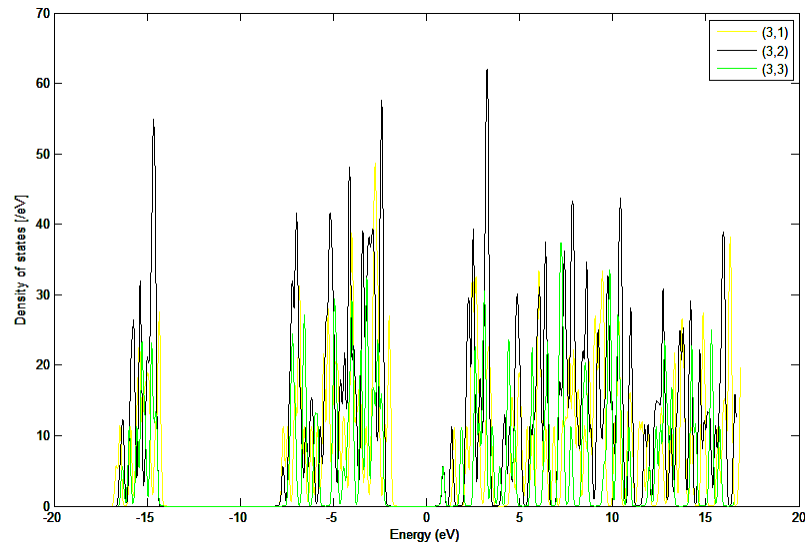

Fig 6. Density of states of $(3,1),(3,2),(3,3) \mathrm{GaN}$ nanotubes

Figure 6 shows the density of states of $(3,1),(3,2)$ and $(3,3)$ configurations plotted. As it can be seen, the density of states peaks for $(3,2)$ among the three configurations. (3,2) nanotube has denser states but the distribution of states follows similar pattern for all the three nanotubes.

Electron density profile can be obtained from the simulations. It has been found that the density is discrete unlike Carbon nanotubes, where the electron density is continuous. The Fermi level of (n, 0) GaN nanotubes are the highest among all the $(n, m)$ GaN.

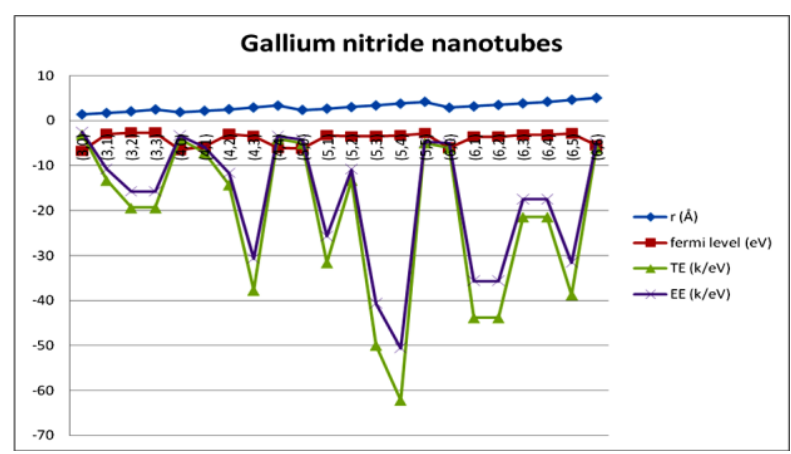

Fig. 7 Radius, Fermi level, total energy and electrostatic energy of various GaN nanotube configurations

Compared to Boron Nitride and Carbon nanotubes of same configuration, Gallium Nitride nanotubes have higher energy in most cases.

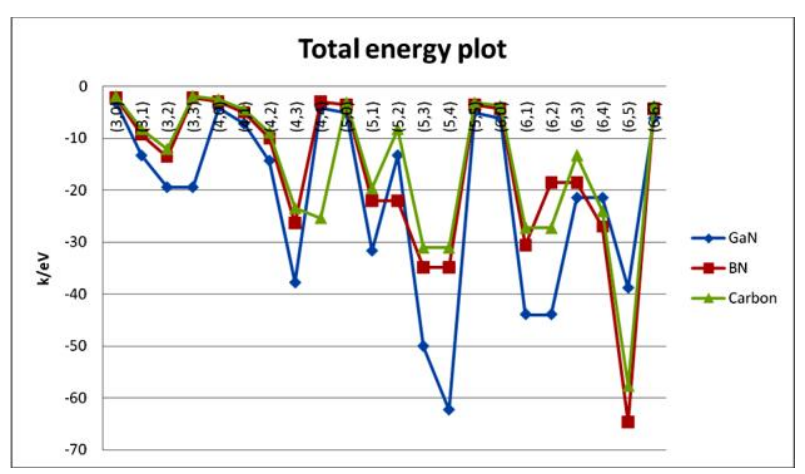

Fig. 8 Comparison of total energy of various configurations of Gallium Nitride, Boron Nitride and Carbon nanotubes. 


\subsection{Gallium Nitride nanotube transistor}

In transistor simulation, we have considered a $(5,5) \mathrm{GaN}$ nanotube with regions around it defining the source, drain, gate and dielectric. This simulation was performed to understand how density of states, transmission spectrum and electron density changes compared to nanotube itself simulated on the same platform.

It has been found that density of states is higher in GaN nanotube transistor compared to Carbon nanotube but less than Boron Nitride nanotube transistor. The band structure of the device was also semiconducting. There was not much difference regarding electron density. The nanotube transistor was simulated using quantum concepts of a one-dimensional transistor [21] in MATLAB. The parameters for the design are tabulated in the table 2 .

The parameters are based on the simulations performed before for Carbon nanotube transistors with a change in the density of states, Fermi level and some parameters which belongs to GaN nanotubes [21].

Table 2: List of parameters used for GaN nanotube transistor modeling

\begin{tabular}{|c|c|c|}
\hline S.No & Parameter & Value \\
\hline 1. & Configuration & ( $\mathrm{n}, \mathrm{m}, \mathrm{c}$-direction) \\
\hline 2. & Length & $10 \times 10^{-9} \mathrm{~m}$ \\
\hline 3. & Width & $1 \times 10^{-6} \mathrm{~m}$ \\
\hline 4. & Thickness & $1 \times 10^{-9} \mathrm{~m}$ \\
\hline 5. & Temperature & $300 \mathrm{~K}$ \\
\hline 6. & Dielectric constant & 4 \\
\hline 7. & Input voltage range & $0-10 \mathrm{~V}$ \\
\hline 8. & Gate voltage & $0.5 \mathrm{~V}$ \\
\hline 9. & Fermi level & Based on nanotube \\
\hline 10. & Density of states & Based on nanotube \\
\hline 11. & Lattice constant & $1.76 \times 10^{-10} \mathrm{~m}$ \\
\hline 12. & Bond length & $0.90 \times 10^{-10} \mathrm{~m}$ \\
\hline 13. & Escape velocity & $1 \times 10^{7} \mathrm{~ms}^{-1}$ \\
\hline 14. & $\begin{array}{l}\text { Electrochemical potential } \\
\text { (contacts) }\end{array}$ & 0.9 (palladium) \\
\hline 15. & Effective mass & 0.25 \\
\hline
\end{tabular}

As it can be seen in figure 6, the density of states in Gallium Nitride nanotubes is entirely random compared to density of states of Carbon nanotubes.

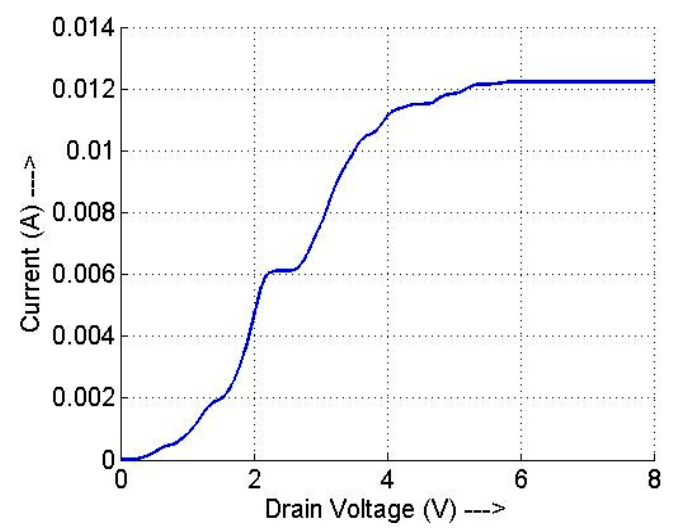

Fig 9. Current versus drain voltage of GaN nanotube transistor
Figure 9 shows the current-voltage plot obtained from the model. The saturation current value is $12 \times 10^{-3} \mathrm{~A}$ which is good compared to previously designed Carbon nanotube transistor (FETTOY) which gave a current of $30 \times 10^{-6} \mathrm{~A}$ [24]. The variations in the transition region of current are due to unevenly distributed density of states of Gallium Nitride nanotubes.

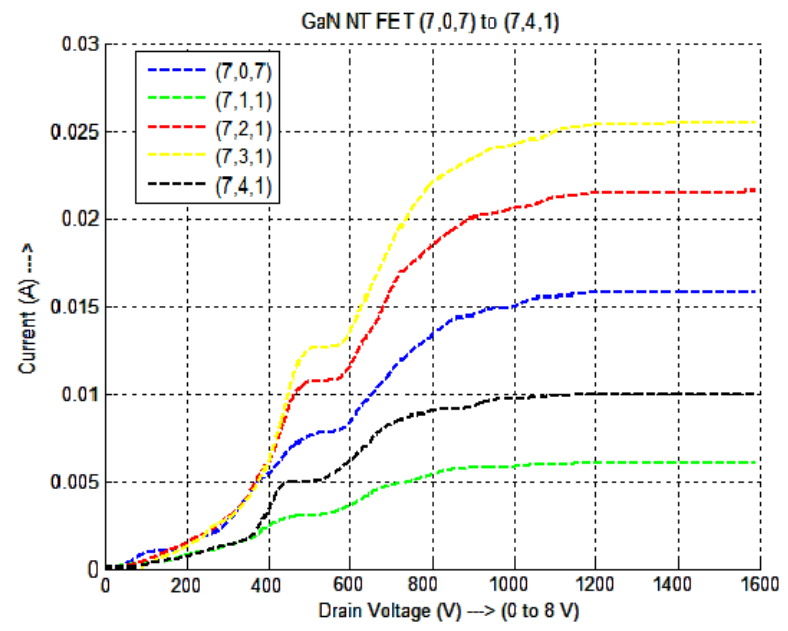

Fig 10. Current versus drain voltage of GaN nanotube transistor with $(7,0)$ to $(7,4) \mathrm{GaN}$ nanotube channels

The variations in the transition region of current in transistors made from $(7,0)$ to $(7,4) \mathrm{GaN}$ nanotubes as shown in figure 10 are consistent with FET characteristics.

\section{CONCLUSION}

In this work, we have evaluated Gallium Nitride nanotubes for its efficiency as a transistor channel. The band gaps are $2.5-$ $3.5 \mathrm{eV}$ for the nanotubes of radius less than $6 \AA$ Á. Fermi function is highest for armchair configuration which decides the number of electrons. It can be inferred that (n, n-1) GaN nanotubes have higher density of states compared to all $(\mathrm{n}, \mathrm{m})$ $\mathrm{GaN}$ nanotubes with similar distribution of states. It has been found from simulation studies that the energy of $(n, n-1) \mathrm{GaN}$ nanotubes are higher compared to all $(\mathrm{n}, \mathrm{m}) \mathrm{GaN}$ nanotubes and can be declared as most stable among all (n, m) nanotubes. GaN nanotube transistor exhibited characteristics similar to conventional field effect transistor with a mild variations corresponding to bizarre density of states in the $\mathrm{GaN}$ nanotubes. GaN nanotube is designed using the same procedure as that of Carbon nanotubes. Palladium contacts had been used for simulation, as materials with lower work function can be used.

\section{REFERENCES}

[1] Philip L. Taylor, Olle Heinonen, "A Quantum Approach to Condensed Matter Physics," Cambridge University Press, 2002.

[2] Aaron Franklin, Mathieu Luisier, Shu jen Han, George Tulevski, Chris Breslin, Lynne Gignac, Mark Lundstrom and Wilfried Haensch, "Sub-10 nm Carbon nanotube transistor," Nano letters, Vol. 12, pp 758-762, 2012.

[3] V. V. Pokropivnyi, "Nanostructured Materials - NonCarbon Nanotubes (Review): Types and Structure," Powder Metallurgy and Metal Ceramics, Vol. 40, Nos. $11-12,2001$

[4] David P. Williams, Stefan Schulz, Aleksey D. Andreev, and Eoin P. O'Reilly, Member, IEEE, "Theory of GaN 
Quantum Dots for Optical Applications,” IEEE Journal Of Selected Topics In Quantum Electronics, pp 1092 1103, Vol. 15, No. 4, July 2009.

[5] Marti D, Bolognesi CR, Cordier Y, Chmielowska M \& Ramdani M. "RF Performance of $\mathrm{AlGaN} / \mathrm{GaN}$ HighElectron-Mobility Transistors Grown on Silicon (110)," Applied Physics Express, Vol. 4, 2011.

[6] Joshua Goldberger, Rongrui He, Yanfeng Zhang, Sangkwon lee, Haoquan Yan, Heon Jin Choi, Peidong Yang, "Single Crystal Gallium Nitride nanotubes," Nature, Vol. 442, pp 599-602, 2003.

[7] N.G. Chopra, R.J. Luyken, K.Cherrey, V.H. Crespi, M.L. Cohen, S.G. Louie, A.Zettl, "Boron-Nitride nanotubes," Science, Vol. 269, 1995.

[8] Min Zhang, Zhong Min Su, Li Kai Yan, Yong Qing Qiu, Gua Hua Chen, Rong Shun Wang, "Theoretical interpretation of different nanotube morphologies among Group III (B, Al, Ga) Nitrides," Chemical Physics Letters, Vol. 408, pp 145 - 149, 2005.

[9] Lee, S. M., Lee, Y. H., Hwang, Y. G., Elsner, J. and Porezag, D., Frauenheim, "Stability and Electronic Structure of GaN Nanotubes from Density-Functional Calculations," Physical Review B, Vol. 60, pp 77887791, 1999.

[10] Yeau Ren Jeng, Ping Chi Tsai and Te Hua Fang, "Tensile Deformation of Tubular Structures of Nitride-based Nanotubes: Brittle and Weak Behavior", Tamkang Journal of Science and Engineering, Vol. 8, pp 191 $195,2005$.

[11] Atomiztix tool kit - Virtual Nanolab software from Quantum wise.

[12] R.G. Parr and W. Yang, "Density-Functional Theory of Atoms and Molecules," Oxford, 1989.

[13] Hohenberg and Kohn, "Inhomogeneous electron gas," Physical Review B, Vol. 136, pp 864-871, 1964.
[14] Kohn and Sham, "Self-consistent Equations including Exchange and Correlation effects," Physical Review Archive, Vol. 140, pp 1133-1138, 1965.

[15] J.P. Perdew, Alex Zunger, "Self-interaction correction to density-functional approximations for many-electron systems," Physical Review B, Vol. 23, pp 5048-5079, 1981.

[16] Richard Wilson, "GaN market due for power boost," Electronics Weekly, March 2012

[17] M.C. Lu, Y.L. Chueh, L.J. Chen, L.J. Chou, H.L. Hsiao and An Ba Yang, "Synthesis and Formation Mechanism of Gallium Nitride Nanotubular Structure," Electrochemical and Solid State Letters, Vol. 8, pp 153 155, 2005.

[18] S. B. Thapa, F. Scholz, "Epitaxial Growth of ZnO-GaN Hetero-Nanorods and GaN Nanotubes," Annual Report 2008, Institute of Optoelectronics, Ulm University, pp 35 $-40,2008$.

[19] Shang Chao Hung, Yan Kuin Su, Te hua Fang, Shoou Jinn Chang and Liang Wen Ji, "Buckling instabilities in $\mathrm{GaN}$ nanotubes under uniaxial compression," IOP Nanotechnology, pp 2203-2208, 2005.

[20] Enling Li, Xiqiang Wang, Liping Hou, Danna Zhao, Yuanbin Dai, Xuewen Wang, "Study on the Electronic Transport Properties of zigzag GaN nanotubes,” Journal of Physics: Conference Series, Vol. 276, 2011.

[21] Supriyo Datta, "Quantum Transport: From atom to transistor," Cambridge University Press, 2005.

[22] J.M. Seminario, "Molecular and Nano Electronics: Analysis, Design and Simulation," Elsevier, 2007.

[23] Phaedon Avouris, Joerg Appenzeller, Richard Martel, Shalom Wind, "Carbon Nanotube Electronics", Proceedings of the IEEE, Vol 91, Vo. 11, November 2003.

[24] A. Rahman, J. Guo, S. Datta and M. Lundstrom, "Theory of Ballistic Nanotransistors," IEEE Transactions on Electron Devices, Vol. 50, pp 1853-1864, 2003. 